Invited Paper

\title{
Non-invasive Investigations of a Wall Painting using Optical Coherence Tomography and Hyperspectral Imaging
}

\author{
Haida Liang ${ }^{*}$, Rebecca Lange ${ }^{\mathrm{a}}$, Helen Howard ${ }^{\mathrm{b}}$, Jane Spooner ${ }^{\mathrm{c}}$ \\ ${ }^{a}$ School of Science and Technology, Nottingham Trent University, Nottingham NG11 8NS, UK \\ ${ }^{\mathrm{b}}$ National Gallery, Trafalgar Square, London WC2N 5DN, UK \\ ${ }^{c}$ Historic Royal Palaces, 4B The Casemates, The Tower of London, EC3N 4AB, UK
}

\begin{abstract}
Multispectral and hyperspectral imaging are efficient methods of measuring spectral reflectance at high spatial resolution. This non-invasive technique has been applied to the imaging of paintings over the last 20 years. PRISMS (Portable Remote Imaging System for Multispectral Scanning) was designed specifically for imaging wall paintings. Optical Coherence Tomography (OCT) is a low coherence interferometric technique capable of fast non-invasive imaging of subsurface microstructure. This paper shows the first application of in situ OCT imaging of a wall painting. The combination of PRISMS and OCT gives information on the varnish and paint layer structure, pigment identification, the state of degradation of the paint and varnish layers and informing curators on the painting schemes and techniques.
\end{abstract}

Keywords: multispectral imaging, hyperspectral imaging, remote imaging, infrared imaging, pigment identification, optical coherence tomography, non-invasive imaging

\section{INTRODUCTION}

Optical Coherence Tomography (OCT) applications to heritage science is currently an active research area ${ }^{1,2}$. Direct comparisons between OCT virtual cross-section images and real cross-sections of paint samples showed the effectiveness of OCT imaging. Apart from the non-invasive examination of the stratigraphy of paint and varnish layers, OCT has also been shown to be the most sensitive technique for revealing preparatory sketches or underdrawings beneath paint layers owing to its high dynamic range, depth selection capabilities and resolution.

In this paper, we demonstrate the effectiveness of OCT imaging of wall paintings through a case study of a recent in situ OCT examination of a $14^{\text {th }}$-century English wall painting in the Byward Tower of the Tower of London. Unlike crosssections of paint samples viewed under a microscope, OCT virtual cross-section images do not have color information. However, a combination of OCT images of paint layers and multispectral/hyperspectral imaging of the same area can yield structural and spectral information non-invasively. Color information is then easily derived from spectral reflectance measurements.

The wall painting was also imaged with a hyperspectral/multispectral imaging system - PRISMS (Portable Remote Imaging System for Multispectral Scanning) which was designed specifically for the in situ imaging of wall paintings ${ }^{3,4}$. High resolution multispectral/hyperspectral images (sub-mm resolution) spanning the wavelength range of $400 \mathrm{~nm}$ to $1700 \mathrm{~nm}$ can be obtained in situ from a distance of less than a meter to over ten meters. It has been applied to the imaging of wall paintings ${ }^{4}$, easel paintings ${ }^{5}$ and manuscripts ${ }^{6}$ for pigment identification, detecting preparatory sketches as well as other 'hidden' information such as areas of past intervention.

The Tower of London wall painting depicts a Crucifixion scene with flanking saints. The figure of St Michael the Archangel is the most intact (Fig. 1). It is one of the most well-preserved medieval murals in the UK and the only surviving medieval painted interior at the Tower of London. The style of the painting suggests it dates to the 1390s.

*haida.liang@ntu.ac.uk; phone 441158488056

O3A: Optics for Arts, Architecture, and Archaeology III, edited by Luca Pezzati, Renzo Salimbeni, Proc. of SPIE Vol. 8084, 80840F · (c) 2011 SPIE · CCC code: 0277-786X/11/\$18 · doi: 10.1117/12.890088 
However, it is not known who painted it or why it was there. A research project is underway to try and answer these and other questions.

Curatorial questions regarding the painting are: 1) is there an underdrawing? 2) what is the varnish and paint layer structure? Was the mural painted in one scheme or was it originally painted with just the green and gold background and then the figures were added at a much later date?

Routine examination of wall paintings is carried out by taking tiny samples to examine under a microscope. To identify pigments in various layers, the sample is prepared and polished to examine the cross-section. The pigments can be identified visually according to their color and shape. For detailed analysis of the material composition, routine analytical chemistry techniques such as SEM, FTIR, XRD etc. are used. The disadvantage of the methods is that it is invasive and conservation ethics restricts sampling to regions of damage or edges of a painting, hence it is difficult to know if the tiny sample is representative of other visually similar regions on the painting.

The advantage of non-invasive and non-contact investigations over invasive techniques is that it is possible to examine any region on an object giving a global rather than a restricted view of the composition of an object. With non-invasive methods, the entire object can be examined, which is impossible with invasive techniques without damaging the objects.

An effective method of investigation is by combining non-invasive techniques with in-depth analysis of selected samples. The non-invasive techniques can be used for global investigations to identify strategic areas for sampling. The invasive chemical analysis methods have the advantage of being able to give more specific and detailed information about the material composition. There are however cases where it is not possible to carry out any invasive investigation.

The paper shows how the combined information from OCT and PRISMS can be used to assist the understanding of the painting scheme and technique, the identification of the material and layer structures.

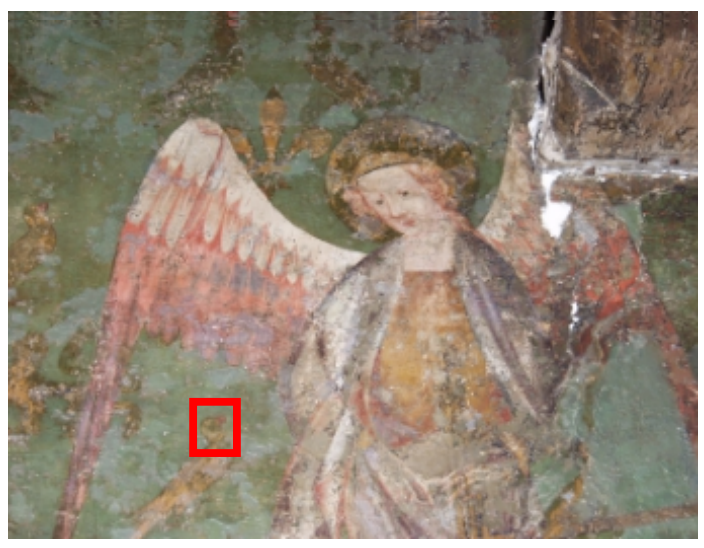

Figure 1. The figure of St Michael in the Tower of London mural: the red box shows the head of the popinjay where detailed OCT and PRISMS imaging were conducted. Photo credit: Stephen Paine with permission from Historic Royal Palaces.

\section{REMOTE SPECTRAL IMAGING}

PRISMS is designed for the in situ imaging of wall paintings which can be found on the walls or ceilings of large architectural spaces such as cathedrals or in narrow spaces such as tomb corridors ${ }^{3,4}$. It is therefore designed to be flexible and able to image at high resolution (i.e. sub-mm resolution) both at close range and remote distances ( $>3.5 \mathrm{~m}$ and up to $10 \mathrm{~m}$ or more). For flexibility and portability, the instrument is designed to be modular. Remote imaging is achieved by using a small telescope, and for close range imaging a lens is used. The visible filtering system was designed to be of low budget and it has 10 interference filters covering the range from $400 \mathrm{~nm}-900 \mathrm{~nm}$ with bandwidth of $40 \mathrm{~nm}$ (the $880 \mathrm{~nm}$ filter has a bandwidth of $70 \mathrm{~nm}$ ). In the short wave infrared (SWIR) range of $900-1700 \mathrm{~nm}$, an AOTF 
spectrograph was used along with an InGaAs camera ${ }^{7}$. The ATOF can tune to any wavelength in the range and with adjustable bandwidth from $10 \mathrm{~nm}$ to $150 \mathrm{~nm}$.

The painting was imaged in situ from a distance of $\sim 6 \mathrm{~m}$ by placing PRISMS on the ground. No scaffolding was necessary and the spatial resolution of the images was $\sim 50 \mu \mathrm{m}$ per pixel.

A test version of the PRISMS system was taken to the Tower of London to image the painting in 2008 in the visible range $^{4}$ and more recently with the full system. Near infrared images were taken around St Michael's eye to reveal the presence of any underdrawing. The original $880 \mathrm{~nm}$ image did not reveal any underdrawing. However, it is known that most paints become more transparent at longer wavelengths, hence it was worth trying with SWIR imaging. Images were taken between $1000-1600 \mathrm{~nm}$ with $150 \mathrm{~nm}$ bandwidth to maximize the chance of detection since some paints are more transparent in specific spectral windows. However, no underdrawings were detected up to $1700 \mathrm{~nm}$. Infrared imaging is successful in detecting underdrawings only if the spectral response of the drawing is different from the background. For example, we can not exclude the possibility of a red ochre underdrawing.

The other objective of PRISMS imaging was pigment identification. For comparison with the unknown spectra, the $\sim 50$ reference samples of historic artists' pigments in linseed oil were also imaged through PRISMS. An algorithm for identifying both single pigments and pigment mixtures similar to those described in one of our previous papers ${ }^{5}$ was followed, where pigment mixtures were predicted from Kubelka-Munk theory. Figure 2 shows that the red paint is identified with vermilion and the yellow paint on the collar of the popinjay is identified with yellow ochre. However, the lighter yellow over the head of the popinjay has a spectrum distinct from the yellow paint on the collar of the popinjay (Fig. 2). The spectrum of the general green background and the greens on the popinjay were compared (Fig. 2) and the spectra of the greens on the popinjay appears to be suppressed in the blue compared with the background greens. Figure 2 shows that while a paint mixture with verdigris and lead white in linseed oil provided the best fit from the reference set of historic artists' pigments, it is by no means a good fit. It was initially thought that the difference might be due to a layer of old yellowed varnish.

Some tiny samples were taken from the painting and had their cross-sections prepared and examined under an optical microscope and with SEM/EDX. Some of these samples were also analyzed with FTIR and GC-MS. The conclusion from the chemical analysis was that the green was a mixture of verdigris and lead white in linseed oil. There are two possible explanations for why the spectrum of the green pigment did not match the reference verdigris and lead white mixture: 1) there is a layer of yellowed varnish that suppressed the blue part of the spectrum and therefore shifted the peak to longer wavelengths; 2) the paint has altered over time and shifted the peak of the spectrum to the red. The first hypothesis can be tested by imaging the painting with an OCT.

\section{COMBINED OCT AND SPECTRAL IMAGING}

OCT imaging can provide two types of information: 1) layer structure of paint and varnish; 2) the scattering and absorption properties of paint and varnish layers. A $930 \mathrm{~nm}$ Fourier domain OCT with depth resolution of $\sim 6.5 \mu \mathrm{m}$ and transverse resolution of $\sim 9 \mu \mathrm{m}$ was used to image parts of the painting in order to help answer some of the questions highlighted by the multispectral imaging results. Unfortunately, with OCT imaging we still needed scaffolding as the OCT objective needs to be $\sim 1 \mathrm{~cm}$ from the painting. The OCT probe head was attached to a motorized XYZ micrometer stage in order to scan extended areas.

The OCT virtual cross-section image in Fig. 3 shows that the red paint was painted above the yellow collar which in turn appears to be painted on top of the varnish layer followed by the green paint layer on the popinjay. In addition, the yellow that is painted roughly on the head of the popinjay (y1 in Fig. 2) appears to be very thin and transparent (at least at $930 \mathrm{~nm}$ ) such that the varnish layer and the green paint below the varnish can be seen through the yellow layer (Fig. 3). This suggests that perhaps the spectrum from this yellow region should be interpreted as a layer of yellow paint above the varnished green paint. A trial model mixture of the spectrum from the yellow on the collar (y2) and the varnished green spectrum (g2) appears to be consistent with the measured spectrum of the yellow from the region y1 (Fig. 2). 
The exposed green on the popinjay is covered with a layer of varnish of an average thickness of $\sim 20 \mu \mathrm{m}$ assuming that the refractive index of the varnish is $\sim 1.5$ (Fig. 3). However, most of the green background is no longer covered with varnish. A region on the green background that was not covered by varnish was identified ( $\mathrm{g} 3$ in Fig. 2) and its spectrum was then compared with the reference spectrum of verdigris and lead white mixture in Figure 2. It is clear that the green spectrum does not fit the reference spectrum of verdigris and lead white. This leaves us with the conclusion that the green paint and/or its binding medium has altered over time, which is most likely due to a reaction between the pigments and the binding medium.
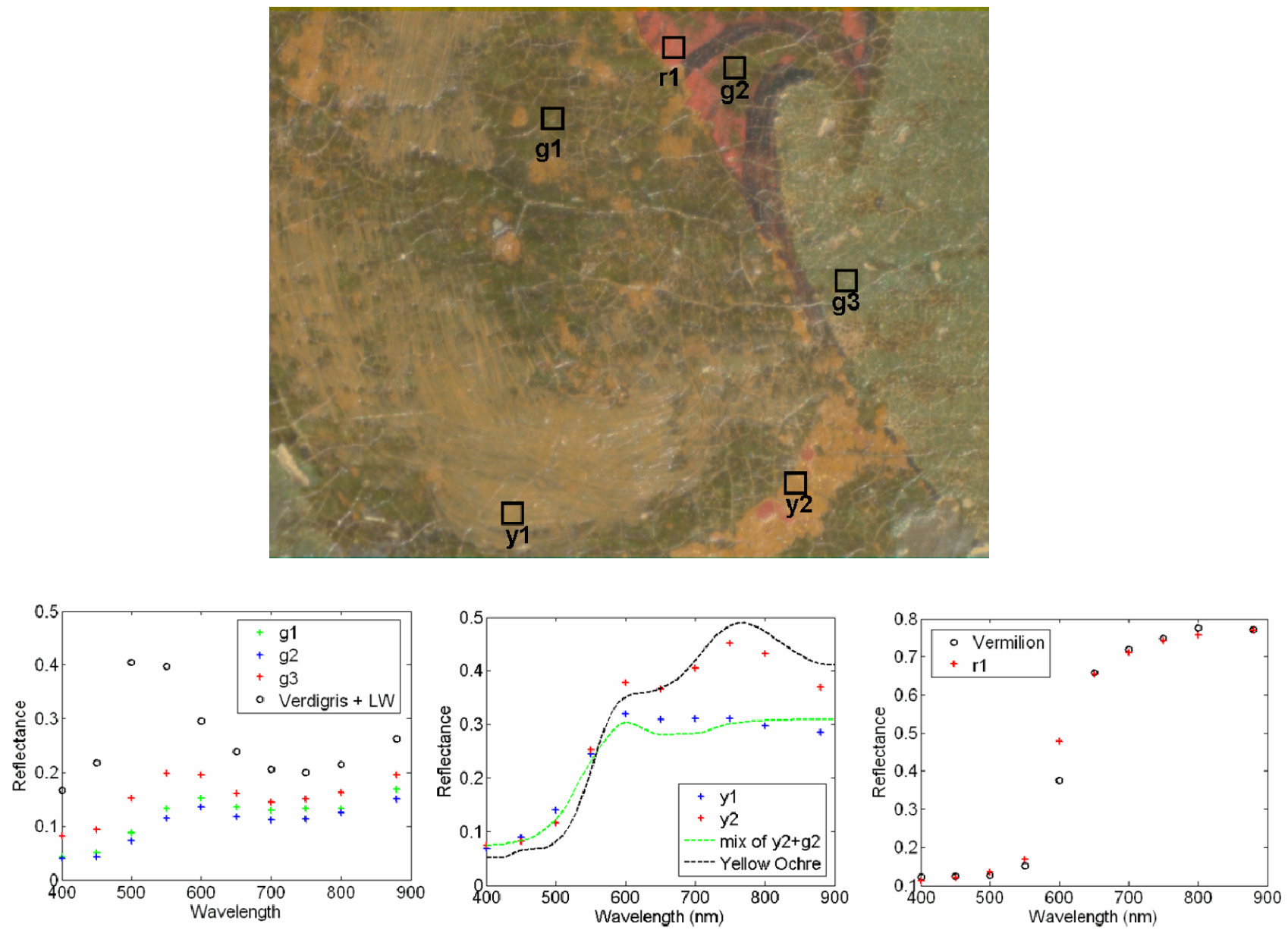

Figure 2. TOP: area of the popinjay imaged in one field of view of PRISMS; BOTTOM Left: PRISMS derived spectral reflectance of the various greens ( $\mathrm{g} 3$ is the background green region without varnish, g1 and g2 are greens on the popinjay with varnish on top) and a reference spectrum of verdigris and lead white; Middle: PRISMS derived spectra of the two types of yellows and reference spectra of yellow ochre and a model fit of a combination of the yellow (from the yellow collar) and the green (from the popinjay); Right: PRISMS derived spectrum of the red paint and a reference sample of vermilion in linseed oil.

We can test in more detail whether or not the green paint on the popinjay is just the same green paint in the background but covered with a yellowed varnish. Since the OCT can measure the thickness of the varnish at each point where the painting was imaged with PRISMS, the extinction coefficient of the yellowed varnish can be found through a fit to Beer's law $R(\lambda)=R_{0}(\lambda) \exp (-2 k(\lambda) t)$ where $R$ and $R_{0}$ are the reflectance of the green paint with a layer of yellow varnish on top and without varnish respectively, $k$ is the absorption coefficient and $t$ is the thickness of the varnish. Alternatively, the effect of a yellowed varnish of a similar thickness on a 50 year old test painting was used to 
simulate the effect of such a varnish on the spectral reflectance of the unvarnished green paint. Figure 4 shows that the simulated varnished green paint is consistent with those measured on the popinjay and hence confirming that the green on the popinjay is the same as the green paint on the background. It is can be seem in the OCT image in Fig. 4 that the light is scattered within the varnish layer, which is an indication that the varnish has degraded. A layer of new varnish should be transparent and should not scatter light.

The depth intensity profiles of the red and yellow paints were also compared with reference vermilion and yellow ochre (in linseed oil) paints respectively and found that the scattering and absorption properties were similar and thus confirming the detection from earlier spectral imaging results. The depth intensity profiles of the green paint did not agree with either reference verdigris oil paint or verdigris and lead white mixture in linseed oil.
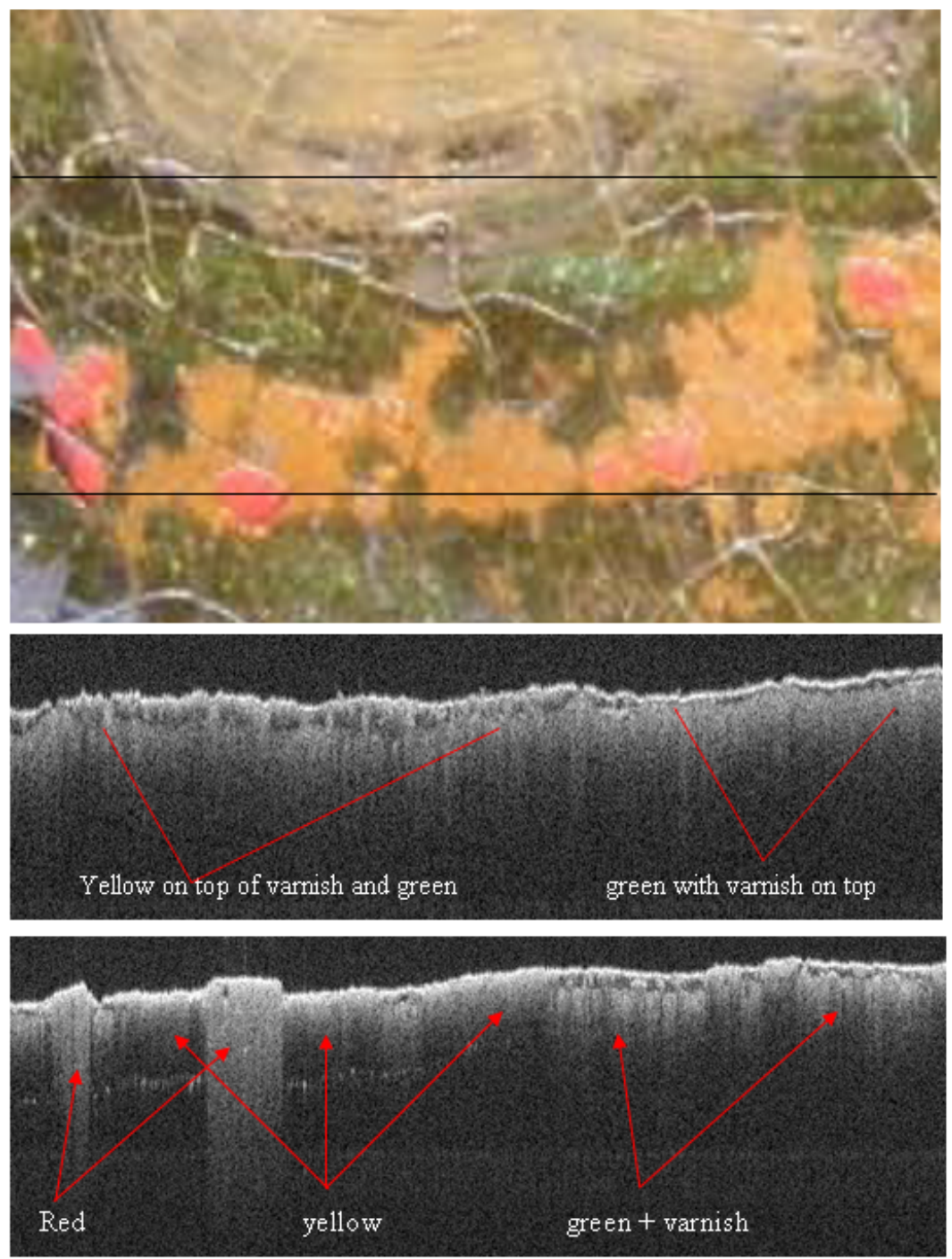

Figure 3. TOP: color image around the head of the popinjay covering an area scanned by the OCT; MIDDLE: an OCT virtual crosssection along the top horizontal black line shown in the color image; BOTTOM: an OCT virtual cross-section along the bottom horizontal black line shown in the color image (the bright dots half way down the image on the left are artifacts). The OCT crosssection images are $8 \mathrm{~mm}$ by $0.81 \mathrm{~mm}$. 


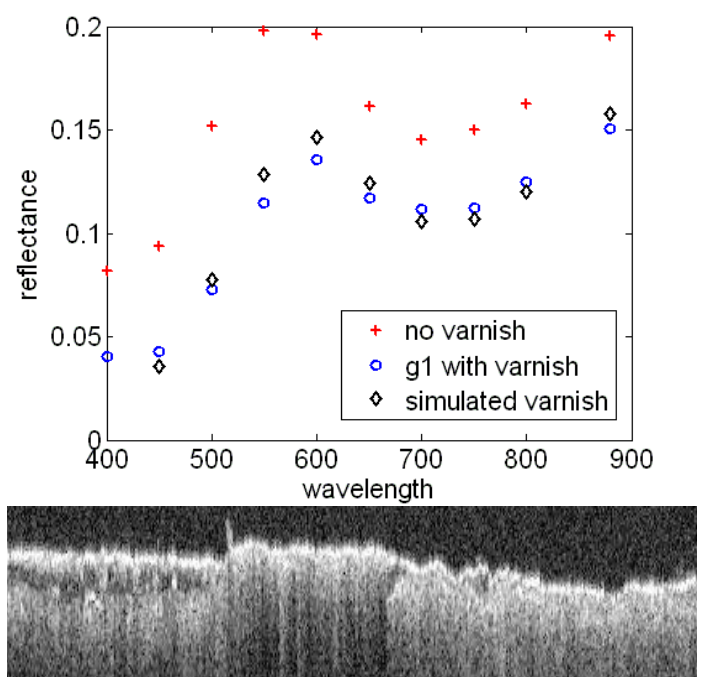

Figure 4 TOP: spectral reflectance of the background green shown in red crosses (g3 in Fig. 2), a region of the green (with yellowed varnish on top) on the popinjay shown in blue circles (g1 in Fig. 2) and the simulated spectrum of g3 (i.e. unvarnished green background) covered by a layer of yellow varnish similar to that found over g1 (black diamonds); BOTTOM: zoomed in OCT virtual cross-section image showing on the left a varnished green on the popinjay, to the right the unvarnished green background and in the middle the black outline of the popinjay. The image scale is $4.1 \mathrm{~mm}$ by $0.32 \mathrm{~mm}$.

\section{CONCLUSIONS}

The combination of the two non-invasive imaging techniques, multispectral imaging using PRISMS and OCT yields valuable information that can not be obtained with either one of the techniques alone or with invasive sampling methods since sampling can only be restricted to areas of damage and the size of samples are less than $1 \mathrm{~mm}$. OCT provides measurements of the scattering and absorption properties of the paints which gives additional confirmation of the pigment identification. OCT probing of the amount of scattering within the varnish layer also provides an indication of the state of degradation of the varnish. An accurate mapping of the varnish thickness using the OCT provides a means of quantitative virtual varnish removal when combined with PRISMS multispectral images.

\section{ACKNOWLEDGEMENTS}

This work was supported by a UK Engineering and Physical Sciences Research Council (EPSRC) grant (EP/E016227/1) and a EPSRC and Arts and Humanities Research Council (AHRC) grant under the Science and Heritage program (AH/H032665/1). We would like to thank Simon Godber, Tom Vajzovic for contribution to PRISMS control software, Kafing Keita for assisting with the first field trip and initial data analysis, Elizabeth Bemand and Sammy Cheung for assisting with the second field trip, Borislava Peric and Marika Spring (National Gallery London) for some of the reference paint-outs, colleagues at Historic Royal Palaces for various assistance, John Cupitt for maintaining the VIPS/nip software (http://www.vips.ecs.soton.ac.uk/), Chris Pannell and Jon Ward at Gooch \& Housego for the AOTF spectrograph.

\section{REFERENCES}

[1] Targowski, P., Góra, M., Wojtkowski, M., "Optical Coherence Tomography for Artwork Diagnostics", Laser Chemistry, vol. 2006, doi:10.1155/2006/35373, 11 pages, (2006) 
[2] Liang, H., Peric, B., Hughes, M., Podoleanu, A. Gh., Spring, M., and Roehrs, S., "Optical Coherence Tomography in archaeological and conservation science - a new emerging field," Proc. SPIE 7139, 713915 (2008)

[3] Liang, H., Keita, K. and Vajzovic, T., "PRISMS: A portable multispectral imaging system for remote in situ examination of wall paintings," Proc. SPIE 6618, 661815 (2007).

[4] Liang, H., Keita, K., Vajzovic, T., Zhang, Q., "PRISMS: remote high resolution in situ multispectral imaging of wall paintings," International Council of Museums, Committee for Conservation (ICOM-CC) Triennial Conference Vol. I, 353-358 (2008).

[5] Liang, H., Keita, K., Peric, B. and Vajzovic, T., "Pigment identification with optical coherence tomography and multispectral imaging," Proc. OSAV'2008, The 2nd International Topical Meeting on Optical Sensing and Artificial Vision, St Petersburg Russia 2008, 33-42 (2008)

[6] Liang, H., "Advanced Optical Imaging Methods for Investigating Manuscripts," [The Technical Study of Books and Manuscripts as Artefacts - research questions and analytical solutions], S. Neate, D. Howell, R. Ovenden, A.M. Pollard eds, (BAR International Series 2209, Oxford 2011), 55-66 (2011).

[7] Liang, H., Keita, K., Pannell, C., Ward, J., "A SWIR hyperpsectral imaging system for art history and art conservation”, IX CONGRESO NACIONAL DEL COLOR, Alicante, Spain 2010, 189-192 (2010) 